\title{
Ceftolozane/tazobactam for the treatment of multidrug resistant Pseudomonas aeruginosa: experience from the Balearic Islands
}

\author{
Manuel Díaz-Cañestro ${ }^{1}$ - Leonor Periañez ${ }^{2}$ - Xavier Mulet ${ }^{3}$ - M. Luisa Martin-Pena ${ }^{1}$ - Pablo A. Fraile-Ribot ${ }^{3}$. \\ Ignacio Ayestarán ${ }^{4}$. Asunción Colomar ${ }^{4}$ - Belén Nuñez ${ }^{5} \cdot$ Maria Maciá $^{5}$ • Andrés Novo ${ }^{6}$. Vicente Torres ${ }^{7}$ - Javier Asensio ${ }^{1}$. \\ Carla López-Causapé ${ }^{3} \cdot$ Olga Delgado $^{2}$ - José Luis Pérez ${ }^{3}$ - Javier Murillas ${ }^{1} \cdot$ Melchor Riera $^{1} \cdot$ Antonio Oliver $^{3}$
}

Received: 19 July 2018 / Accepted: 15 August 2018 / Published online: 23 August 2018

(C) Springer-Verlag GmbH Germany, part of Springer Nature 2018

\begin{abstract}
A prospective, descriptive observational study of consecutive patients treated with ceftolozane/tazobactam in the reference hospital of the Balearic Islands (Spain), between May 2016 and September 2017, was performed. Demographic, clinical, and microbiological variables were recorded. The later included resistance profile, molecular typing, and whole genome sequencing of isolates showing resistance development. Fifty-eight patients were treated with ceftolozane/tazobactam. Thirty-five (60.3\%) showed respiratory tract infections, 21 (36.2\%) received monotherapy, and 37 (63.8\%) combined therapy for $\geq 72 \mathrm{~h}$, mainly with colistin (45.9\%). In $46.6 \%$ of the patients, a dose of $1 / 0.5 \mathrm{~g} / 8 \mathrm{~h}$ was used, whereas $2 / 1 \mathrm{~g} / 8 \mathrm{~h}$ was used in $41.4 \%$. In 56 of the cases (96.6\%), the initial Pseudomonas aeruginosa isolates recovered showed a multidrug resistant (MDR) phenotype, and 50 of them (86.2\%) additionally met the extensively drug resistant (XDR) criteria and were only susceptible colistin and/or aminoglycosides (mostly amikacin). The epidemic high-risk clone ST175 was detected in 50\% of the patients. Clinical cure was documented in 37 patients $(63.8 \%)$ and resistance development in $8(13.8 \%)$. Clinical failure was associated with disease severity (SOFA), ventilator-dependent respiratory failure, XDR profile, high-risk clone ST175, negative control culture, and resistance development. In 6 of the 8 cases, resistance development was caused by structural mutations in AmpC, including some mutations described for the first time in vivo, whereas in the other 2, by mutations in OXA-10 leading to the extended spectrum OXA14. Although further clinical experience is still needed, our results suggest that ceftolozane/tazobactam is an attractive option for the treatment of MDR/XDR P. aeruginosa infections.
\end{abstract}

Keywords Ceftolozane/tazobactam $\cdot$ Multidrug resistant $\cdot$ Pseudomonas aeruginosa

Electronic supplementary material The online version of this article (https://doi.org/10.1007/s10096-018-3361-0) contains supplementary material, which is available to authorized users.

Manuel Díaz-Cañestro

manuel.diaz@ssib.es

Antonio Oliver

antonio.oliver@ssib.es

1 Servicio de Medicina Interna-Infecciosas, Hospital Universitari Son Espases, Instituto de Investigación Sanitaria Illes Balears (IdISBa), Palma de Mallorca, Spain

2 Servicio de Farmacia Hospitalaria, Hospital Universitari Son Espases, Instituto de Investigación Sanitaria Illes Balears (IdISBa), Palma de Mallorca, Spain

3 Servicio de Microbiología y Unidad de Investigación, Hospital Universitari Son Espases, Instituto de Investigación Sanitaria Illes Balears (IdISBa), Palma de Mallorca, Spain
4 Servicio de Medicina Intensiva, Hospital Universitari Son Espases, Instituto de Investigación Sanitaria Illes Balears (IdISBa), Palma de Mallorca, Spain

5 Servicio de Pneumología, Hospital Universitari Son Espases, Instituto de Investigación Sanitaria Illes Balears (IdISBa), Palma de Mallorca, Spain

6 Servicio de Hematología, Hospital Universitari Son Espases, Instituto de Investigación Sanitaria Illes Balears (IdISBa), Palma de Mallorca, Spain

7 Servicio de Reanimación, Hospital Universitari Son Espases, Instituto de Investigación Sanitaria Illes Balears (IdISBa), Palma de Mallorca, Spain 


\section{Introduction}

Antibiotic resistance is currently considered one of the largest public health threats. Increasing infections with multidrug resistant (MDR) microorganisms and the shortage of antibiotic therapeutic arsenal in the last years compromise the selection of adequate treatments, leading to an increase in morbidity and mortality rates $[1,2]$. There are many causes that favor the selection and dissemination of antibiotic resistance, but the inappropriate and indiscriminate use of antibiotics together with deficient infection control measures are likely those providing a wider margin for reducing this global threat. MDR Enterobacteriaceae, Acinetobacter baumannii, and Pseudomonas aeruginosa are the pathogens for which the situation is considered critical by the World Health Organization (WHO).

Ceftolozane/tazobactam is the combination of a new cephalosporin with the $\beta$-lactamase inhibitor tazobactam, which shows a high in vitro activity against gram-negative bacteria including MDR and extensively drug resistant (XDR) Pseudomonas aeruginosa [3]. Moreover, ceftolozane/ tazobactam has been shown to conserve activity against $P$. aeruginosa strains that have developed resistance to all other available beta-lactams, mainly due to its high stability against the hydrolysis by the chromosomal cephalosporinase AmpC $[4,5]$. Additionally, ceftolozane/tazobactam also exhibits certain activity against extended spectrum beta-lactamase (ESBL)-producing Enterobacteriaceae [3].

Ceftolozane/tazobactam has been approved for the treatment of complicated intra-abdominal infections [6] (cIAI) and for the treatment of complicated urinary tract infections including acute pyelonephritis [7] (UTI) and is currently under evaluation for the treatment of nosocomial pneumonia. Due to the growing prevalence of MDR/XDR P. aeruginosa, showing resistance to all first-line agents, including available $\beta$ lactams and fluoroquinolones, ceftolozane/tazobactam could be a promising alternative to existing options that currently include more toxic and likely less effective antibiotics, such as aminoglycosides and polymyxins. However, the current experience is still limited to a few reported cases [8-11] with a 21patient cohort from a hospital in Pittsburgh, USA and a 35patient cohort from 6 hospitals from Houston being the largest series published so far $[12,13]$. Additionally, we described some years ago the mechanisms leading to in vitro ceftolozane/tazobactam resistance development [14], and a few cases of treatment failure due to resistance development have been recently reported [12].

In May 2016, ceftolozane/tazobactam was approved by the Pharmacy and Therapeutics Committee of Balearic Public Health System with the following indications: (i) urinary and intra-abdominal infections caused by ESBL-producing Enterobacteriaceae or MDR P. aeruginosa, (ii) other infections caused by MDR P. aeruginosa in the absence of appropriate therapeutic alternatives according to the patient's characteristics, and (iii) in P. aeruginosa bacteremia or pneumonia in patients colonized or admitted in areas showing high prevalence of MDR P. aeruginosa. The last two were off-label indications motivated by the lack of appropriate alternatives. Moreover, since the approval of ceftolozane/tazobactam, its use has been monitored under a specific antimicrobial stewardship program. Thus, the main objectives of this study were to evaluate the use of ceftolozane/tazobactam in a large cohort (58 patients) from a third-level hospital and to analyze the efficacy of this new therapeutic option for the treatment of MDR P. aeruginosa infections, including clinical and microbiological endpoints, with a particular focus in the analysis of resistance development during treatment.

\section{Material and methods}

\section{Patients and setting}

We conducted a prospective, descriptive observational study of consecutive patients treated with ceftolozane/tazobactam for at least $48 \mathrm{~h}$ in the Son Espases University Hospital (HUSE) of Palma de Mallorca, Spain, between May 2016 and September 2017. The study was approved by the research committee of HUSE. HUSE is the reference tertiary care hospital for the Balearic Islands and is equipped with 1020 beds of hospitalization, surgery, intensive care unit, and emergency departments. All the prescriptions in the hospital are made using a computerized physician order entry (CPOE). HUSE follows an antimicrobial stewardship program, run by a team of pharmacists, microbiologists, and infectious diseases clinicians, which provides daily monitoring and treatment advice for infections by MDR pathogens.

\section{Variables and definitions}

Demographic, clinical, and microbiological variables studied included the following: age, sex, underlying diseases, renal replacement therapy, ventilator-dependent respiratory failure, Charlson comorbidity score [15], predictive scale of multiorganic dysfunction (SOFA) [16], type of infection according to CDC definitions, sample of MDR $P$. aeruginosa isolation, characteristics of ceftolozane/tazobactam treatment (empiric/targeted, monotherapy/combined, duration and dose, and whether it was indicated according to recommendation approved by the Pharmacy and Therapeutics Committee of Balearic Public Health System), resistance phenotype, clonal type, and ceftolozane/tazobactam MIC. The primary endpoint was clinical failure defined as persistent signs or symptoms of infection and positive culture after 7 days of treatment. Secondary endpoints included crude 30 days mortality, microbiological cure (negative control culture), colonization by the 
infecting strain, and ceftolozane/tazobactam resistance development.

\section{Susceptibility testing, molecular epidemiology, and resistance mechanisms}

Initial antimicrobial susceptibility was tested by broth microdilution (Microscan ${ }^{\circledR}$ ) for ceftazidime, cefepime, piperacillin-tazobactam, aztreonam, imipenem, meropenem, ciprofloxacin, gentamicin, tobramycin, amikacin, and colistin and with gradient strips (Liofilchem $®$ ) for ceftolozane/tazobactam. EUCAST breakpoints were considered in all cases, and consensus recommendations [17] were used to define MDR and XDR phenotypes. Molecular typing of all P. aeruginosa isolates recovered was performed by pulsed-field gel electrophoresis (PFGE) and multilocus sequence typing (MLST) as described previously [18]. The presence of horizontallyacquired $\beta$-lactamases was evaluated using phenotypic and molecular (PCR followed by sequencing) methods [19]. Intestinal colonization by MDR/XDR $P$. aeruginosa was investigated in most patients through the culture of rectal swabs in selective media before, during, and after the treatment as part of HUSE program for MDR pathogens surveillance.

\section{Characterization of the mechanisms leading to ceftolozane/tazobactam resistance development}

An additional extended broth microdilution panel (Thermofisher) including a whole range of concentrations of all above-tested antipseudomonal agents and ceftazidime/ avibactam was used to further analyze the pairs of ceftolozane/tazobactam susceptible/resistant isolates when resistance development was documented. Moreover, DNA sequence variation between susceptible and resistant pairs of isolates was analyzed through whole genome sequencing as previously described [19]. Briefly, indexed paired-end libraries were generated from genomic DNA using a commercial library preparation kit (Nextera XT DNA Library Preparation Kit; Illumina) and sequenced on an Illumina MiSeq benchtop sequencer with a MiSeq Reagent Kit (version 3; Illumina Inc., USA). Obtained paired-ended reads were aligned to $P$. aeruginosa PAO1 reference genome, and sequence variation was further analyzed for the 146 chromosomal genes related to antimicrobial resistance. Additionally, the presence of horizontally acquired resistance determinants was further explored using online databases (https://cge.cbs.dtu.dk// services/ResFinder/). A total of 8 cases of resistance development were documented. Resistance mechanisms for the first 5 have already been published [20], and therefore this work adds the characterization of the 3 remaining cases.

\section{Statistical analysis}

Quantitative variables were compared using the MannWhitney $U$ test. Chi-square $\left(\chi^{2}\right)$ was used to determine the association between factors and clinical cure or resistance development. In all cases, a $p$ value $<0.05$ was considered statistically significant. Multivariate logistic regression of independent risk factors associated to clinical cure was performed. Multivariate analysis was performed using a stepwise backward method. Crude and adjusted odds ratio (OR) are shown. Comparison of the expected and observed frequencies by the Hosmer-Lemeshow goodness-of-fit test $(p$ value $=0.117)$ and by ROC curve (AUC $=0.903 ; p<0.001$ ) indicated a good fit for the model. All statistical analyses were performed using GraphPad Prism 5 or IMB SPSS® Statistics v22 software.

\section{Results}

Up to 58 patients were treated with ceftolozane/tazobactam at HUSE from May 2016 to December 2017. The demographic, clinical, and microbiological variables studied are shown in Table 1. Sixteen of the patients (27.6\%) were admitted to the ICU when treatment was initiated, $35(60.3 \%)$ showed respiratory tract infections, and $10(17.2 \%)$ showed urinary tract infections. Twenty-one (36.2\%) received ceftolozane/ tazobactam monotherapy and $37(63.8 \%)$ combined therapy for $\geq 72 \mathrm{~h}$, mainly with colistin (45.9\%), amikacin (21.6\%), or tobramycin $(18.9 \%)$.

In $46.6 \%$ of the patients, a ceftolozane/tazobactam dose of $1 / 0.5 \mathrm{~g} / 8 \mathrm{~h}$ was used, whereas the $2 / 1 \mathrm{~g} / 8 \mathrm{~h}$ dose was used in $41.4 \%$. Of the 25 patients with respiratory infections, without renal insufficiency $(\mathrm{CrCl}>60 \mathrm{~mL} / \mathrm{min}), 3$ received the doses approved by the FDA of $1 / 0.5 \mathrm{~g} / 8 \mathrm{~h}$, whereas 22 received doses of $2 / 1 \mathrm{~g} / 8 \mathrm{~h}$. On the other hand, of 9 patients with respiratory infection who received continuous renal replacement therapy (CRRT), 7 were treated with doses of $1 / 0.5 \mathrm{~g} / 8 \mathrm{~h}$ and 2 with doses of $0.5 / 0.25 \mathrm{~g} / 8 \mathrm{~h}$. The rest of patients with CRRT received doses of $0.5 / 0.25 \mathrm{~g} / 8 \mathrm{~h}$ except for two patients (urinary, skin, and soft tissue infection) who received 1/0.5 g/ $8 \mathrm{~h}$. Two patients with moderate renal insufficiency $(\mathrm{CrCl} 30$ $50 \mathrm{~mL} / \mathrm{min}$ ) and urinary infection were treated with doses of $0.5 / 0.25 \mathrm{~g} / 8 \mathrm{~h}$ and those with respiratory infection with a dose of $1 / 0.5 \mathrm{~g} / 8 \mathrm{~h}$. The two patients with oteoarticular infection received doses of $2 / 1 \mathrm{~g} / 8 \mathrm{~h}$.

Treatment was considered indicated according to recommendations approved by the Balearic Island Pharmacy Committee in $91.4 \%$ of the patients. In 56 of the cases (96.6\%), the recovered $P$. aeruginosa isolates showed an MDR phenotype, and 50 of them (86.2\%) additionally met the XDR criteria and were only susceptible colistin and/or aminoglycosides (mostly amikacin). The median ceftolozane/tazobactam MIC was $2 \mathrm{mg} / \mathrm{L}$, and the widespread 
Table 1 Demographic, clinical, and microbiological characteristics

\begin{tabular}{|c|c|}
\hline Variable & Number or value \\
\hline Age in years $($ mean $\pm \mathrm{SD})$ & $60.8 \pm 14.5$ \\
\hline Sex male, $[n(\%)]$ & $35(60.3 \%)$ \\
\hline \multicolumn{2}{|l|}{ Underlying diseases } \\
\hline Immunosuppressed & $7(12.06 \%)$ \\
\hline Stem cell transplant & $2(3.44 \%)$ \\
\hline Chemotherapy & $2(3.44 \%)$ \\
\hline AIDS & $2(3.44 \%)$ \\
\hline Organ transplant & $1(1.72 \%)$ \\
\hline Neutropenic $(<1000)$ & $2(3.44 \%)$ \\
\hline Cardiovascular disease & $19(32.75 \%)$ \\
\hline Cystic fibrosis & $2(3.44 \%)$ \\
\hline \multicolumn{2}{|l|}{ Chronic obstructive } \\
\hline Pulmonary disease & $17(29.31)$ \\
\hline Malignancy & $19(32.75 \%)$ \\
\hline Charlson index [median (R)] & $4(0-11)$ \\
\hline Ventilator-dependent respiratory failure & $19(32.75 \%)$ \\
\hline SOFA [median (R)] & $3(0-18)$ \\
\hline \multicolumn{2}{|l|}{ Hospital ward $[n(\%)]$} \\
\hline Pneumology & $15(25.9 \%)$ \\
\hline Intensive Care Unit & $16(27.6 \%)$ \\
\hline Internal Medicine & $8(13.8 \%)$ \\
\hline Oncohematological & $5(8.62 \%)$ \\
\hline Surgical & $5(8.62 \%)$ \\
\hline Reanimation & $4(6.89 \%)$ \\
\hline Others & $5(8.62 \%)$ \\
\hline \multicolumn{2}{|l|}{ Source of infection $[n(\%)]$} \\
\hline Respiratory & $35(60.3 \%)$ \\
\hline Urinary & $10(17.2 \%)$ \\
\hline Intra-abdominal & $4(6.9 \%)$ \\
\hline Bacteriemia & $3(5.2 \%)$ \\
\hline Osteoarticular & $2(3.4 \%)$ \\
\hline Other & $4(6.9 \%)$ \\
\hline \multicolumn{2}{|l|}{ Origin $[n(\%)]$} \\
\hline Nosocomial & $54(93.1 \%)$ \\
\hline Community & $4(6.9 \%)$ \\
\hline \multicolumn{2}{|l|}{ Type of treatment $[n(\%)]$} \\
\hline Targeted & $53(91.4 \%)$ \\
\hline Semiempirical & $4(6.9 \%)$ \\
\hline Empirical & $1(1.7 \%)$ \\
\hline \multicolumn{2}{|l|}{ Treatment } \\
\hline Combined & $37(63.8 \%)$ \\
\hline Colistin & $17(45.9 \%)$ \\
\hline Amikacin & $8(21.6 \%)$ \\
\hline Tobramycin & $7(18.9 \%)$ \\
\hline Monotheraphy & $21(36.2 \%)$ \\
\hline Indicated treatment $[n(\%)]$ & $53(91.4 \%)$ \\
\hline Duration of treatment (days, mean $\pm \mathrm{SD}$ ) & $11.4 \pm 6.2$ \\
\hline $\begin{array}{l}\text { Renal failure replacement therapy at the time of } \\
\text { initiation of C-T }\end{array}$ & $15(25.8 \%)$ \\
\hline
\end{tabular}

Table 1 (continued)

\begin{tabular}{ll}
\hline Variable & Number or value \\
\hline Posology $[n(\%)]$ & \\
$1-0.5 \mathrm{~g} / 8 \mathrm{~h}$ & $27(46.6 \%)$ \\
$2-1 \mathrm{~g} / 8 \mathrm{~h}$ & $24(41.4 \%)$ \\
$0.5-0.25 \mathrm{~g} / 8 \mathrm{~h}$ & $7(12.1 \%)$ \\
Type of sample $[n(\%)]$ & \\
Bronchial aspirate & $20(34.5 \%)$ \\
Sputum & $12(20.7 \%)$ \\
Urine & $9(15.5 \%)$ \\
Blood & $7(12.1 \%)$ \\
Others & $10(17.2 \%)$ \\
Ceftolozane/tazobactam MIC $[$ median $(\mathrm{R})]$ & $2(0.75-6)$ \\
Resistance profile $[n(\%)]$ & \\
XDR & $50(86.2 \%)$ \\
MDR & $6(10.3 \%)$ \\
Pretreatment colonization $[n(\%)]$ & $29(50 \%) / \mathrm{ND}(18)$ \\
Negativized colonization $(29)[n(\%)]$ & $4(13.79 \%)$ \\
Molecular typing ST175 $[n(\%)]$ & $29(50 \%)$ \\
Resistance development $[n(\%)]$ & $8(13.8 \%)$ \\
Negative control culture $[n(\%)]$ & $21(70 \%)$ \\
Clinical cure $[n(\%)]$ & $(\mathrm{unknown} 28)$ \\
Death at 30 days $[n(\%)]$ & $37(63.8 \%)$ \\
& $16(27.6 \%)$ \\
\hline &
\end{tabular}

epidemic high-risk clone ST175 was detected in 50\% of the patients. Clinical cure was documented in 37 patients (63.8\%) and resistance development in 8 (13.8\%).

\section{Analysis of factors associated with clinical failure}

A comparative univariate analysis using clinical failure as dependent variable was performed (Table 2). Significant differences were documented for disease severity (SOFA), ventilator-dependent respiratory failure, XDR profile, highrisk clone ST175, negative control culture, and resistance development. Additionally, a multivariate analysis of factors associated with ceftolozane/tazobactam clinical failure was performed (Table 3). While patients infected by ST175 or documented resistance development showed a 3.8 and 6.6 times higher risk of clinical failure, respectively, the multivariate analysis only identified ventilator-dependent respiratory failure as independent risk factor.

\section{Analysis of factors associated with resistance development}

A comparative univariate analysis using resistance development as dependent variable was also performed (Table 1S), and only clinical cure and negative control culture yielded significant differences. Nevertheless, although statistical 
Table 2 Univariate analysis of factors associated with clinical failure

\begin{tabular}{|c|c|c|c|}
\hline Variable & $\begin{array}{l}\text { Clinical } \\
\text { failure } \\
(N=21)\end{array}$ & $\begin{array}{l}\text { Clinical cure } \\
(N=35)\end{array}$ & $\begin{array}{l}p \\
\text { value }\end{array}$ \\
\hline Age in years (mean $\pm \mathrm{SD})$ & $65.4 \pm 11.7$ & $62 \pm 16.0$ & 0.219 \\
\hline Sex male, $[n(\%)]$ & $13(61.9 \%)$ & $21(60 \%)$ & 0.888 \\
\hline Charlson index [median (R)] & $5(0-11)$ & $4(0-11)$ & 0.177 \\
\hline Ventilator-dependent respiratory failure & $15(71.42 \%)$ & $4(11.42)$ & 0.000 \\
\hline SOFA [median (R)] & $5(1-18)$ & $2(0-18)$ & 0.000 \\
\hline Hospital ward $[n(\%)]$ & & & 0.007 \\
\hline Pneumology & $3(14.3 \%)$ & $11(31.4 \%)$ & \\
\hline Intensive Care Unit & $11(52.4 \%)$ & $4(11.4 \%)$ & \\
\hline Internal Medicine & $1(4.8 \%)$ & $7(20 \%)$ & \\
\hline Other & $6(28.6 \%)$ & $13(37.2 \%)$ & \\
\hline Source of infection $[n(\%)]$ & & & 0.217 \\
\hline Respiratory & $15(71.4 \%)$ & $18(51.4 \%)$ & \\
\hline Urinary & $2(9.5 \%)$ & $8(22.9 \%)$ & \\
\hline Intra-abdominal & $1(4.8 \%)$ & $3(8.6 \%)$ & \\
\hline Bacteriemia & $2(9.5 \%)$ & $1(2.9 \%)$ & \\
\hline Osteoarticular & $0(0 \%)$ & $2(5.8 \%)$ & \\
\hline Other & $1(4.8 \%)$ & $3(8.6 \%)$ & \\
\hline Origin $[n(\%)]$ & & & 0.168 \\
\hline Nosocomial & $21(100 \%)$ & $32(91.4 \%)$ & \\
\hline Community & $0(0.0 \%)$ & $3(8.6 \%)$ & \\
\hline Type of treatment $[n(\%)]$ & & & 0.127 \\
\hline Targeted & $20(95.2 \%)$ & $31(88.6 \%)$ & \\
\hline Semiempirical & $0(0.0 \%)$ & $4(11.4 \%)$ & \\
\hline Empirical & $1(4.8 \%)$ & $0(0 \%)$ & \\
\hline Treatment & & & 0.618 \\
\hline Combined & $14(66.6 \%)$ & $21(60 \%)$ & \\
\hline Monotherapy & $7(33.3 \%)$ & $14(40 \%)$ & \\
\hline Posology $[n(\%)]$ & & & 0.620 \\
\hline $1-0.5 \mathrm{~g} / 8 \mathrm{~h}$ & $7(33-33 \%)$ & $16(45.71 \%)$ & \\
\hline $2-1 \mathrm{~g} / 8 \mathrm{~h}$ & $11(52.38 \%)$ & $15(42.85 \%)$ & \\
\hline $0.5-0.25 \mathrm{~g} / 8 \mathrm{~h}$ & $3(14.28 \%)$ & $4(11.42 \%)$ & \\
\hline $\begin{array}{l}\text { Renal failure replacement therapy at the time of initiation of } \\
\text { C-T }\end{array}$ & $8(38.09 \%)$ & $6(17.14 \%)$ & 0.080 \\
\hline Duration of treatment (days, mean $\pm \mathrm{SD}$ ) & $9.95 \pm 5.38$ & $13.21 \pm 6.42$ & 0.177 \\
\hline Ceftolozane/tazobactam MIC [median (R)] & $2.00(1-6)$ & $2.00(0.75-4)$ & 0.138 \\
\hline Resistance profile $[n(\%)]$ & & & 0.045 \\
\hline $\mathrm{XDR}$ & $21(100 \%)$ & $29(82.82 \%)$ & \\
\hline MDR & $0(0.0 \%)$ & $6(17.14 \%)$ & \\
\hline Pretreatment colonization $[n(\%)]$ & $14(66.6 \%)$ & $14(47.1 \%)$ & 0.142 \\
\hline Negativized colonization (29) $[n(\%)]$ & $1(4.8 \%)$ & $3(8.6 \%)$ & 0.310 \\
\hline Molecular typing ST175 $[n(\%)]$ & $15(75 \%)$ & $14(47.1)$ & 0.022 \\
\hline Resistance development $[n(\%)]$ & $6(28.6 \%)$ & $2(5.7 \%)$ & 0.018 \\
\hline Negative control culture $[n(\%)]$ & $4(30.7 \%)$ & $17(100 \%)$ & 0.000 \\
\hline Death at 30 days $[n(\%)]$ & $16(76.19 \%)$ & $0(0.0 \%)$ & 0.000 \\
\hline
\end{tabular}

significance was not reached, it is worthy to mention that in most of the cases of resistance development $(6 / 8,75 \%)$, a respiratory infection was involved and was colonized before the start of treatment $(7 / 887.5 \%)$ (Table $1 \mathrm{~S})$. Likewise, there was a tendency for a higher initial ceftolozane/tazobactam MIC (median 3 vs $2 \mathrm{mg} / \mathrm{L}$ ). On the other hand, no association was found for the use of ceftolozane/tazobactam in monotherapy or in combination. In fact, 7 of the 8 patients in which 
Table 3 Risks factors for clinical failure

\begin{tabular}{llr}
\hline Variable & \multicolumn{1}{l}{$\begin{array}{l}\text { Crude OR } \\
\text { (Lower-Upper) }\end{array}$} & $p$ value \\
\hline Ventilator-dependent respiratory failure* & $19.4(4.743-79.149)$ & $<0.001$ \\
SOFA & $6.6(2.5-17.6)$ & $<0.001$ \\
Ceftolozane/tazobactam resistance development & $6.6(1.2-36.6)$ & 0.031 \\
Clone involved ST175 & $3.8(1.2-12.0)$ & 0.026 \\
Respiratory tract infection & $2.6(0.8-8.4)$ & 0.099 \\
Duration of treatment & $0.9(1.0-0.8)$ & 0.073 \\
Ceftolozane/tazobactam MIC & $1.9(1.0-3.6)$ & 0.067 \\
Renal failure replacement therapy & $3.0(0.86-10.3)$ & 0.086 \\
\hline
\end{tabular}

*Multivariate analysis only identified ventilator-dependent respiratory failure as independent risk factor resistance development was documented received combined therapy, mainly with colistin ( 4 cases) or amikacin (2 cases).

\section{Characterization of mechanisms leading to ceftolozane/tazobactam resistance development}

Resistance development was documented in $8(13.8 \%)$ of the cases. Table 4 shows the antimicrobial susceptibility profiles and main resistance mechanisms documented for the 8 pairs of ceftolozane/tazobactam susceptible/resistant $P$. aeruginosa clinical isolates studied, of which the first 5 (as indicated in the table) have been already published [20]. Both isolates in each pair were confirmed to belong to the same clonal type through PFGE (not shown). In 6 of the 8 cases, ceftolozane/ tazobactam resistance development involved mutations leading to the structural modification of $\mathrm{AmpC}$; in 5 of them, the infecting strain belonged to ST175 and the remaining one to ST235. In addition to the previously characterized T96I, E247K, and $\Omega$ loop deletions [20], two new AmpC mutations are reported in vivo for the first time in this work: a new amino acid replacement in the key residue E247 (E247G) and the F147L mutation, previously shown to be involved in ceftolozane/tazobactam resistance in in vitro evolution experiments [14]. Finally, in the other 2 cases, the infecting strain belonged to ST179, and ceftolozane/tazobactam resistance development involved a mutation in the narrow spectrum enzyme OXA-10 leading to the extended spectrum OXA-14.

\section{Discussion}

MDR, and particularly XDR, $P$. aeruginosa is among the pathogens for which the introduction of new effective antibiotics is considered critical by the WHO. Indeed, too frequently, the only available susceptible agents are the polymyxins and/or some aminoglycosides, which are considered more toxic and likely less effective than $\beta$-lactams or fluoroquinolones for most types of infections. Thus, the introduction of new $\beta$-lactam combinations, such as ceftolozane/ tazobactam or ceftazidime/avibactam, active against many XDR strains, should alleviate to some extend this concerning scenario [21]. While ceftolozane/tazobactam is not active against strains producing most acquired carbapenemases, it has been shown to conserve susceptibility against $P$. aeruginosa strains resistant to all other available $\beta$-lactams through chromosomal mutations, mainly due to its high stability against the hydrolysis by the chromosomal cephalosporinase AmpC [4, 5, 22, 23]. Thus, the usefulness of ceftolozane/tazobactam for the treatment of XDR $P$. aeruginosa infections should be conditioned by the prevalence of carbapenemases which varies significantly worldwide [24]. A recent multicenter (including our hospital) study performed in Spain showed that ceftolozane/tazobactam was susceptible in close to $70 \%$ of XDR P. aeruginosa isolates and that ceftolozane/tazobactam resistance correlated well with the production of acquired carbapenemases [22].

With 58 patients included, ours is the largest published cohort and mostly included infections by XDR strains only susceptible to colistin and/or some aminoglycosides (mostly amikacin), in addition to ceftolozane/tazobactam, and thus likely representing the scenario in which this novel $\beta$-lactam combination is most needed. Therefore, our work complements the findings from other recent, smaller cohorts of patients with MDR $P$. aeruginosa infections treated with ceftolozane/tazobactam [12]. Clinical cure was documented in $63 \%$ of the cases, similar although slightly lower than the rates $(71 \%)$ reported for the 21-patient cohort of Haidar et al. (2017), likely reflecting differences of disease severity, the most potent independent variables associated with clinical failure in both studies. Even if still not approved, the most frequent indication of ceftolozane/tazobactam treatment was nosocomial pneumonia, as occurred in the study by Haidar et al., highlighting the indication gap needed to be filled by ongoing clinical trials. Indeed, most of the patients with respiratory infections not showing renal insufficiency received doses that doubled those approved by the FDA (22/25 88\%) [25] and in patients with renal failure replacement therapy dose of $1 / 0.5 \mathrm{~g} / 8 \mathrm{~h}(7 / 977.7 \%)$ [26]. 


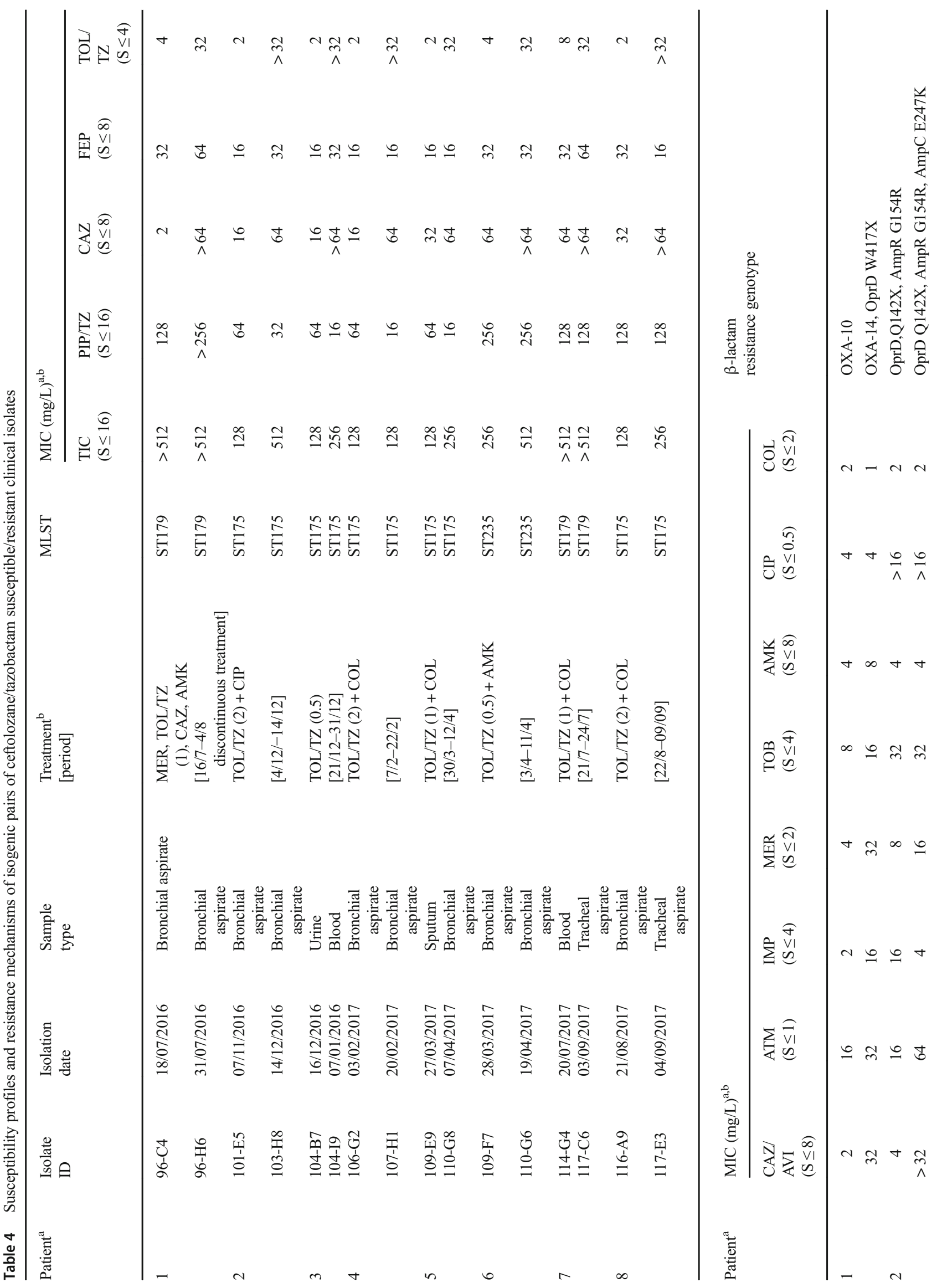




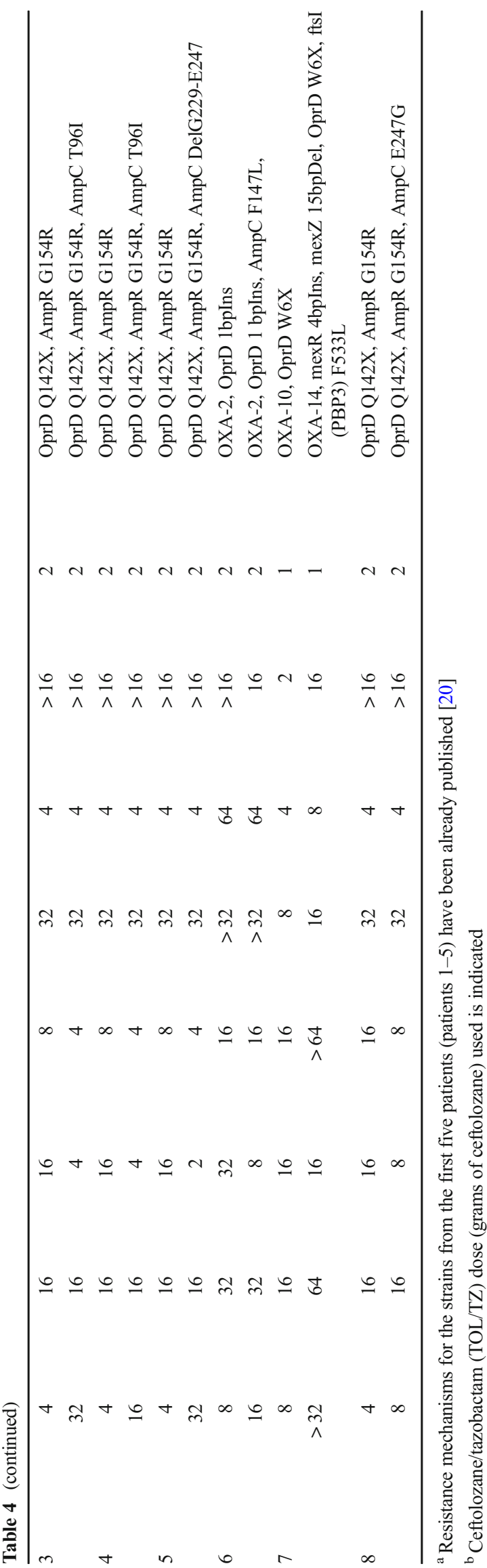

In addition to disease severity markers, clinical failure was found to be associated with several microbiological factors (discussed below), but we failed to demonstrate a significant association with other relevant variables such as the type of infection, the dose of ceftolozane/tazobactam used, or whether it was used in monotherapy or in combination with other active antipseudomonal agent. Nevertheless, larger studies are still needed to fully elucidate these issues. Indeed, the potential benefit of combining active antibiotics for the treatment of $P$. aeruginosa infections is still under controversy [27].

The microbiological variables associated with clinical failure in the univariate analysis were the lack of microbiological eradication, the presence of an XDR phenotype, the involvement of the epidemic ST175 high-risk clone, and the resistance development during therapy. While the association between clinical and microbiological failure seems obvious, the potential impact on clinical outcome of XDR phenotypes or specific epidemic strains is noteworthy. Indeed, the interplay between antibiotic resistance mechanisms, epidemic strains, and virulence is a subject of growing interest [24, 28, 29]. In multivariate analysis, however, ventilator-dependent respiratory failure was the only variable independently associated with clinical failure. Thus, it still needs to be ruled out in larger series whether the association of these microbiological variables with clinical failure could reflect their association with disease severity; however, the association is not expected to be simple or direct, since the XDR phenotype and the ST175 genotype have been recently shown to be associated with a particularly lower virulence [30,31]. In any case, the prevalence of the epidemic high-risk clone ST175 in our series (50\%) was quite similar to the global prevalence of ST175 reported for XDR P. aeruginosa from Spanish hospitals (68\%) [22], and therefore the epidemiological setting should be representative at least at national level.

Finally, the likely most interesting aspect of the study is the evaluation of resistance development during therapy. Indeed, one of the major potential advantages of ceftolozane/tazobactam relays on its lower propensity for resistance development compared to other antipseudomonal agents in vitro, due to its higher stability against $P$. aeruginosa mutational resistance mechanisms [14]. Resistance development was documented in 8 (13.8\%) of the patients, and therefore in a proportion identical to that previously reported by Haidar et al. (3/21, 14.3\%). Thus, coincident findings from both studies, representing two totally different geographical and epidemiological settings, may provide a quite accurate estimation of the likelihood of ceftolozane/tazobactam resistance development during the treatment of MDR/XDR $P$. aeruginosa infections. These values appear to be not lower than those previously reported for other antipseudomonal agents [32], but it should be noted that the MDR/XDR strains targeted with ceftolozane/tazobactam treatments are typically already resistant to all other $\beta$-lactams and contain several resistance mechanisms, particularly noteworthy the hyperproduction of the chromosomal 
$\beta$-lactamase AmpC, the main mechanism of ceftazidime, and other $\beta$-lactams resistance in $P$. aeruginosa [33]. Certainly, resistance development in such challenging strains is expected to be more likely than for wild-type strains. Indeed, consistently with previous in vitro works [14], data from ours and other recent studies confirm that the selection of mutations leading to the structural modification of $\mathrm{AmpC}$, occurring in strains already hyperproducing the cephalosporinase, is the most frequent mechanism of ceftolozane/tazobactam resistance development in vivo. While clinical failure and lack of microbiological eradication were the only variables significantly associated with resistance development in our work, larger studies are needed to rule out the impact of other potentially relevant variables such as the type of infection, combined treatment vs monotherapy, or the initial ceftolozane/tazobactam MIC of the infecting strain. Concerning the later issue, a recent study has evaluated potential dosing solutions for the treatment of infections by strains with MICs above $4 \mathrm{mg} / \mathrm{L}$ [34].

Even if it comprises the largest series published so far, our study is limited by its single center nature and by the lack of a control group, avoiding the possibility of comparison with other available antipseudomonal treatments such as the polymyxins. However, although further clinical experience is still needed, our results, together with those of other recent studies, suggest that ceftolozane/tazobactam is an attractive option for the treatment of MDR/XDR P. aeruginosa infections.

Acknowledgements We are thankful to Pilar Sanchís for her help with the statistical analysis.

Funding This work was supported by the Ministerio de Economía y Competitividad of Spain, Instituto de Salud Carlos III — co-financed by European Regional Development Fund "A way to achieve Europe" ERDF, through the Spanish Network for the Research in Infectious Diseases (RD12/0015 and RD16/0016) and PI15/00088.

\section{Compliance with ethical standards}

Conflict of interest AO has been the recipient of research grants from MSD and has received fees as speaker from MSD.

Ethical approval The study was approved by the research committee of Hospital Universitari Son Espases.

Informed consent Since it was an observational study, informed consent was not required

\section{References}

1. Nathwani D, Raman G, Sulham K, Gavaghan M, Menon V (2014) Clinical and economic consequences of hospital-acquired resistant and multidrug-resistant Pseudomonas aeruginosa infections: a systematic review and meta-analysis. Antimicrob Resist Infect Control $3: 32$
2. Bassetti M, Carnelutti A, Peghin M (2017) Patient specific risk stratification for antimicrobial resistance and possible treatment strategies in gram-negative bacterial infections. Expert Rev AntiInfect Ther 15:55-65

3. Sader HS, Farrell DJ, Flamm RK, Jones RN (2014) Ceftolozane/ tazobactam activity tested against aerobic gram-negative organisms isolated from intra-abdominal and urinary tract infections in European and United States hospitals (2012). J Inf Secur 69:266277

4. Moya B, Zamoramo L, Juan C, Perez JL, Ge Y, Oliver A (2010) Activity of a new cephalosporin, CXA-101 (FR264295), against beta-lactamase-resistant Pseudomonas aeruginosa mutants selected in vitro after antipseudomonal treatment of intensive care unit patients. Antimicrob Agents Chemother 54:1213-1217

5. Moyá B, Beceiro A, Cabot G, Juan C, Zamorano L, Alberti S et al (2012) Pan- $\beta$-lactam resistance development in Pseudomonas aeruginosa clinical strains: molecular mechanisms, penicillinbinding protein profiles, and binding affinities. Antimicrob Agents Chemother 56:4771-4778

6. Solomkin J, Hershberger E, Miller B, Popejoy M, Friedland I, Steenbergen J et al (2015) Ceftolozane/tazobactam plus metronidazole for complicated intra-abdominal infections in an era of multidrug resistance: results from a randomized, double-blind, phase 3 trial (ASPECT-cIAI). Clin Infect Dis 60:1462-1471

7. Wagenlehner FM, Umeh O, Steenbergen J, Yuan G, Darouiche RO (2015) Ceftolozane-tazobactam compared with levofloxacin in the treatment of complicated urinary-tract infections, including pyelonephritis: a randomised, double-blind, phase 3 trial (ASPECTcUTI). Lancet 385:1949-1956

8. Castón JJ, De la Torre A, Ruiz-Camps I, Sorlí ML, Torres V, TorreCisneros J (2017) Salvage therapy with ceftolozane-tazobactam for multidrug-resistant Pseudomonas aeruginosa infections. Antimicrob Agents Chemother 61:2136-2116

9. Xipell M, Bodro M, Marco F, Martínez JA, Soriano A (2017) Successful treatment of three severe MDR or XDR Pseudomonas aeruginosa infections with ceftolozane/tazobactam. Future Microbiol 12:1323-1326

10. Kurtzhalts KE, Mergenhagen KA, Manohar A, Berenson CS (2017) Successful treatment of multidrug-resistant Pseudomonas aeruginosa pubic symphysis osteomyelitis with ceftolozane/tazobactam. BMJ Case Rep

11. Sousa Dominguez A, Perez-Rodríguez MT, Nodar A, MartinezLamas L, Perez-Landeiro A, Crespo Casal M (2017) Successful treatment of MDR Pseudomonas aeruginosa skin and soft-tissue infection with ceftolozane/tazobactam. J Antimicrob Chemother 72:1262-1263

12. Haidar G, Philips NJ, Shields RK, Snyder D, Cheng S, Potoski BA et al (2017) Ceftolozane-Tazobactam for the treatment of multidrugresistant Pseudomonas aeruginosa infections: clinical effectiveness and evolution of resistance. Clin Infect Dis 65:110-120

13. Munita JM, Aitken SL, Miller WR, Perez F, Rosa R, Shimose LA et al (2017) Multicenter evaluation of ceftolozane/tazobactam for serious infections caused by carbapenem-resistant Pseudomonas aeruginosa. Clin Infect Dis 65:158-161

14. Cabot G, Bruchmann S, Mulet X, Zamorano L, Moyá B, Juan C et al (2014) Pseudomonas aeruginosa ceftolozane-tazobactam resistance development requires multiple mutations leading to overexpression and structural modification of AmpC. Antimicrob Agents Chemother 58:3091-3099

15. De Groot V, Beckerman H, Lankhorst GJ, Bouter LM (2003) How to measure comorbidity. A critical review of available methods. J Clin Epidemiol 56:221-229

16. Arts DG, de Keizer NF, Vroom MB, de Jonge E (2005) Reliability and accuracy of sequential organ failure assessment (SOFA) scoring. Crit Care Med 33:1988-1993 
17. Magiorakos AP, Srinivasan A, Carey RB, Carmeli Y, Falagas ME, Giske CG et al (2012) Multidrug-resistant, extensively drugresistant and pandrug-resistant bacteria: an international expert proposal for interim standard definitions for acquired resistance. Clin Microbiol Infect 18:268-281

18. Cabot G, Ocampo-Sosa AA, Domínguez MA, Gago JF, Juan C, Tubau $\mathrm{F}$ et al (2012) Genetic markers of widespread extensively drug-resistant Pseudomonas aeruginosa high-risk clones. Antimicrob Agents Chemother 56:6349-6357

19. Cabot G, López-Causapé C, Ocampo-Sosa AA, Sommer LM, Domínguez MÁ, Zamorano L et al (2016) Deciphering the resistome of the widespread Pseudomonas aeruginosa sequence type 175 international high-risk clone through whole-genome sequencing. Antimicrob Agents Chemother 60:7415-7423

20. Fraile-Ribot PA, Cabot G, Mulet X, Periañez L, Martín-Pena ML, Juan $\mathrm{C}$ et al (2018) Mechanisms leading to in vivo ceftolozane/ tazobactam resistance development during the treatment of infections caused by MDR Pseudomonas aeruginosa. J Antimicrob Chemother 73:658-663

21. Van Duin D, Bonomo RA (2016) Ceftazidime/avibactam and ceftolozane/tazobactam: second-generation $\beta$-lactam/ $\beta$-lactamase inhibitor combinations. Clin Infect Dis 63:234-241

22. Del Barrio-Tofiño E, López-Causapé C, Cabot G, Rivera A, Benito N, Segura C et al (2017) Genomics and susceptibility profiles of extensively drug-resistant Pseudomonas aeruginosa isolates from Spain. Antimicrob Agents Chemother 61:1589-1517

23. Giani T, Arena F, Pollini S, Di Pilato V, D'Andrea MM, Henrici De Angelis L et al (2017) Italian nationwide survey on Pseudomonas aeruginosa from invasive infections: activity of ceftolozane/ tazobactam and comparators, and molecular epidemiology of carbapenemase producers. J Antimicrob Chemother 73:664-671

24. Tato M, Garcia-Castillo M, Bofarull AM, Canton R (2015) In vitro activity of ceftolozane/tazobactam against clinical isolates of Pseudomonas aeruginosa and Enterobacteriaceae recovered in Spanish medical centres: results of the CENIT study. Int J Antimicrob Agents 46:502-510

25. Xiao AJ, Miller BW, Huntington JA, Nicolau DP (2016) Ceftolozane/tazobactam pharmacokinetic/pharmacodynamic- derived dose justifiction for phase 3 studies in patients with nosocomial pneumonia. J Clin Pharmacol 56:56-66

26. Oliver WD, Heil EL, Gonzales JP, Mehrotra S, Robinett K, Saleeb $P$ et al (2016) Ceftolozane-tazobactam pharmacokinetics in a critically ill patient on continuous venovenous hemofiltration. Antimicrob Agents Chemother 60:1899-1901

27. Peña C, Suarez C, Ocampo-Sosa A, Murillas J, Almirante B, Pomar $\mathrm{V}$ et al (2013) Effect of adequate single-drug vs combination antimicrobial therapy on mortality in Pseudomonas aeruginosa bloodstream infections: a post hoc analysis of a prospective cohort. Clin Infect Dis 57:208-216

28. Martínez JL, Baquero F (2002) Interactions among strategies associated with bacterial infection: pathogenicity, epidemicity, and antibiotic resistance. Clin Microbiol Rev 15:647-679

29. Beceiro A, Tomás M, Bou G (2013) Antimicrobial resistance and virulence: a successful or deleterious association in the bacterial world? Clin Microbiol Rev 26:185-230

30. Sánchez-Diener I, Zamorano L, López-Causapé C, Cabot G, Mulet $X$, Peña C et al (2017) Interplay among resistance profiles, high-risk clones, and virulence in the Caenorhabditis elegans Pseudomonas aeruginosa infection model. Antimicrob Agents Chemother 61: $1586-1517$

31. Gómez-Zorrilla S, Juan C, Cabot G, Camoez M, Tubau F, Oliver A et al (2016) Impact of multidrug resistance on the pathogenicity of Pseudomonas aeruginosa: in vitro and in vivo studies. Int $\mathrm{J}$ Antimicrob Agents 47:368-374

32. Carmeli Y, Troillet N, Eliopoulos GM, Samore MH (1999) Emergence of antibiotic-resistant Pseudomonas aeruginosa: comparison of risks associated with different antipseudomonal agents. Antimicrob Agents Chemother 43:1379-1382

33. Zamorano L, Reeve TM, Juan C, Moya B, Cabot G, Vocadlo DJ et al (2011) AmpG inactivation restores susceptibility of pan-betalactam-resistant Pseudomonas aeruginosa clinical strains. Antimicrob Agents Chemother 55:1990-1996

34. Natesan S, Pai MP, Lodise TP (2017) Determination of alternative ceftolozane/tazobactam dosing regimens for patients with infections due to Pseudomonas aeruginosa with MIC values between 4 and $32 \mathrm{mg} / \mathrm{L}$. J Antimicrob Chemother 72:2813-2816 